\section{Estimation of the prevalence at animal level of paratuberculosis in female cattle of Saxony-Anhalt (Germany)}

\author{
Nicolai Denzin, Bernd Gehrmann, \\ Benno Ewert, Holger Rohde \\ State Office of Consumer Protection \\ Saxony-Anhalt, Stendal, Germany
}

\begin{abstract}
Johne's disease (paratuberculosis) is a chronic, untreatable disease of ruminants caused by Mycobacterium avium subspecies paratuberculosis. It leads to economic losses in livestock production but is also suspected to be involved in the pathogenesis of Crohn's disease in humans. In 2007/2008 a study was conducted to estimate the prevalence at animal level of paratuberculosis among the female cattle of Saxony-Anhalt (Germany). In order to keep study costs low and participating farms confidential, no farms were visited to actively draw samples. Instead, animals were preselected in a formal random sampling process and additionally tested by ELISA for paratuberculosis when samples suitable for serology were routinely submitted to the State Office of Consumer Protection Saxony-Anhalt for any diagnostics. Out of 896 samples, 38 tested positive, giving an apparent prevalence of $4.2 \%$. Based on the test quality traits of the ELISA employed in the study (POURQUIER ${ }^{\circledR}$ ELISA Paratuberculosis) the true prevalence was estimated to $6.7 \%$ (95\% CI, 3.0-10.4\%). No spatial clustering of positive results was detected by a scan statistic. Prevalence estimates for age strata tended to show an incline to a maximum at age class of 5 years and a subsequent decline with higher age classes. Estimates tended to show an incline with herd size.
\end{abstract}

\section{Introduction}

Johne's disease (paratuberculosis) is a chronic infectious disease of ruminants caused by Mycobacterium avium subspecies paratuberculosis (MAP). It is characterized by chronic, granulomatous degenerative enteritis that causes intermittent but persistent diarrhoea, progressive weight loss, and eventual death. The disease is untreatable and slowly progressive. ${ }^{1}$ On dairy farms, economic losses occur through reduced milk production, increased cow-replacement costs (pre-mature culling and increased mortality) and lower cull-cow revenues. ${ }^{2}$ Additionally, paratuberculosis might have a zoonotic potential. Since the first description of the similarities of Johne's disease in cattle and Crohn's disease in man in $1913,{ }^{3}$ it has been argued that MAP might also be a cause of Crohn's disease, ${ }^{4-6}$ a chronic inflammatory bowel disease (IBD) which is on the increase in high-income countries. ${ }^{7}$ An association between the presence of MAP and Crohn's disease has been reported in two meta-analyses, 8,9 but causality has not been proven yet.10-12 Infection of humans could theoretically occur through consumption of certain dairy products. MAP is excreted directly into the milk in low numbers but in much higher numbers in the faeces of infected cows, particularly those showing overt clinical signs of Johne's disease. Even with good hygiene some of the infected faeces inevitably gain access to raw milk ${ }^{13}$ and there is evidence that MAP can survive commercial pasteurisation of milk.13-15

These veterinary and potential public health implications of Johne's disease drove the demand of the Ministry of Agriculture and Environment of the federal state SaxonyAnhalt (Federal Republic of Germany) for an estimation of the prevalence of the disease among the state's female cattle. The results from routine diagnosis at the State Office of Consumer Protection were considered as probably biased. Thus, a prevalence study aiming at an unbiased estimation of the animal level prevalence was conducted in 2007/2008. Administrative requirements defined for the study included a minimization of the study costs and an anonymization of the farms from which samples were tested.

A previous serological study of Donat et al. in Saxony, a federal state adjacent to SaxonyAnhalt, led to an estimate of an apparent prevalence of $4.4 \% .16 \mathrm{~A}$ true prevalence was not calculated.

\section{Materials and Methods}

\section{Sampling design}

It was intended to estimate the animal level prevalence in a target population consisting of the female cattle with age 24 months and above (a limitation of the test system; see below) in Saxony-Anhalt. The cattle registered with the national cattle database (see below) comprised the study population. A sample of 1000 specimens drawn from the latter in a formal simple-random sampling process was considered to provide sufficient precision (assumption: expected true prevalence at $8 \%$ calculated according to Shoukri and Edge, 17 see below, accepted absolute error below 4\%) at reasonable costs. The diagnostics were carried
Correspondence: Dr. Nicolai Denzin, Landesamt für Verbraucherschutz Sachsen-Anhalt, Haferbreiter Weg 132-135, 39576 Stendal, Germany.

Tel.+49.3931.631463 - Fax: +49.3931.631103.

E-mail: nicolai.denzin@lav.ms.sachsen-anhalt.de

Key words: Mycobacterium avium subsp. paratuberculosis, cattle, serology, true prevalence.

Acknowledgments: this study was funded by the Disease Control Fund of Saxony-Anhalt, Germany.

Contributions: ND, study design and coordination, statistical analysis, manuscript drafting; BG, diagnostics; $\mathrm{BE}$, fund raising and coordination; HR, software adaptation, programming. All authors read and approved the final manuscript.

Conflict of interest: the authors report no conflicts of interest.

Received for publication: 12 September 2011. Accepted for publication: 14 October 2011.

This work is licensed under a Creative Commons Attribution NonCommercial 3.0 License (CC BYNC 3.0).

(C) Copyright N. Denzin et al., 2010 Licensee PAGEPress, Italy

Veterinary Science Development 2011; 1:e10 doi:10.4081/vsd.2011.e10

out at the State Office of Consumer Protection (LAV) Saxony-Anhalt, Department for Veterinary Medicine, Stendal.

Since the dairy farmer association of Saxony-Anhalt feared a stigmatization of farms included in a paratuberculosis prevalence study or even having animals found testpositive in the study, it was insisting on a study design to keep included farms confidential. This requirement, as well as the general shortage of public funds, led to the decision not to actively visit farms and draw samples for the purpose of the study. Instead, (blood) samples (from cattle) routinely submitted for any examination to the LAV where checked for matches (via the ear tag number) with animals randomly pre-selected (selected on the basis of the national cattle database before the study was launched) from the sampling frame (see below) for inclusion into the study. Samples with matches were then additionally tested for paratuberculosis using a serum ELISA - again limiting costs as compared to faecal culture or faecal polymerase chain reaction (PCR). Submission of preselected samples to the LAV within a reasonable span of time was predominantly ensured by a Bovine Herpes Virus 1 (BHV1) eradication campaign currently run in Saxony-Anhalt and obliging farmers to test cattle for BHV1 at least once per year at the LAV. 


\section{Study period}

The study was carried out between January 2007 and January 2008. Samples were preselected just before study onset in December 2006.

\section{Establishment of the sampling frame}

A list of more than 180,000 animals eligible (only inclusion criteria: female cattle with age 24 months and above) for testing was extracted from the German national cattle database Herkunftssicherungs- und Informationssystem für Tiere (HI-Tier, http://www.hitierde), which incorporates the system for the identification and registration of bovine animals as legally prescribed by Council Regulation (EC) No 820/97 for European Community member states. This data base contains, among other information, the ear tag number, date of birth and registration number of the holding a bovine is currently allocated to, but no geographical co-ordinates. Any changes due to birth, death, slaughter, relocation or trade have to be notified to the system administration within 7 days.

Additionally, livestock holdings have to be geo-coded and administrated by the districts using the software Tierseuchennachrichtensystem (TSN, https://tsn.fli.bund.de) of the Friedrich-Loeffler-Institute, Federal Research Institute for Animal Health, which contains a powerful Geographical Information System (GIS). But the number of animals in the TSN - database of the district is usually only updated a few times per year and no exact dates of birth are recorded, only age classes. Thus, the eligible animals from HI-Tier were linked to the co-ordinates of TSN via the registration number of the holdings in order to determine the spatial distribution of the sampling frame. The sampling frame was broken down in 49 sub frames of a grid of 49 areas (adapted from a $20 \times 20 \mathrm{~km}$ grid applied to Saxony-Anhalt) in Saxony-Anhalt and the density per $\mathrm{km}^{2}$ of sampling units was determined for each area with the help of a GIS.

\section{Selection of the sample}

To improve spatial representativeness of the sample probability proportional to size-sampling ${ }^{18}$ was applied to the 49 areas according to the density of eligible (female cattle with age 24 months and above) animals. The number of samples to be taken from each area was quadruplicated to allow for animals leaving the population (through trade, slaughter, death) before being presented for serological diagnostics at the LAV within the study year. Samples were selected from each sub frame using the random number list generator of Epi Info ${ }^{\mathrm{TM}}$ (Centers for Disease Control \& Prevention, http://wwwn.cdc.gov/epiinfo/).

\section{Sample management}

The Laboratory Information Management System (LIMS) of the LAV is an in-house product based on a Microsoft ${ }^{\mathrm{TM}}$ SQL server. Besides its performance in routine processes the main strong point of the system is its flexibility to users' demands. Adaptations are programmed quickly by the developer who is familiar with the institute's functions and at no additional costs. A feature was integrated into the LIMS to read in the ear tag sample lists of the 49 geographical areas. Whenever there was a match with an ear tag of a sample presented for any serological diagnosis, the sample was marked within the LIMS for an additional testing for paratuberculosis.

Once the predefined number of samples as a share of the overall sample was achieved, the list was closed automatically to avoid surplus testing and expenses.

\section{Diagnostics}

Since the design of the prevalence study did not allow for re-testing or confirmatory testing, emphasis was put on the specificity of the ELISA to be used in the study. Thus, referring to the validation of different tests presented by Köhler et al.,19 samples included in the study were tested with the POURQUIER ${ }^{\circledR}$ ELISA Paratuberculosis (Institut Pourquier, 326 rue de la Galéra, Parc Euromédecine, 34097 Montpellier Cedex 5, France, registration no. BGVV-B-263). The choice of an ELISA as test system excluded cattle below 24 months of age from the target population (see above) because ELISAs will usually not detect such animals if infected, as these have not developed antibodies to MAP. ${ }^{20}$

\section{Calculation of true prevalence and confidence intervals}

The POURQUIER ${ }^{\circledR}$ ELISA Paratuberculosis was, just like all other paratuberculosis ELISAs, an imperfect test. Thus, the true animal level prevalence was calculated from the apparent prevalence according to Rogan and Gladen ${ }^{21}$ assuming a sensitivity (Se) of 0.536 and a specificity (Sp) of 0.993 as reported by Köhler et al..$^{19}$ The latter accuracy estimates were also quoted in the manufacturer's information sheet together with own estimates, which were only slightly lower $(\mathrm{Se}=0.515$, $\mathrm{Sp}=0.990$ ). Meanwhile the Institut Pourquier was taken over by IDEXX, the test was modified (IDEXX Laboratories, Westbrook, Me, USA, IDEXX Paratuberculosis Screening Ab Test, http://www.idexx.com/pubwebresources/ pdf/en_us/livestock-poultry/paratuberculosisscreening-ab-test-sheet.pdf) and the sensitivity was improved.

The confidence interval was calculated according to Shoukri and Edge. ${ }^{17}$ Since the calculation of the Rogan-Gladen-estimator of true prevalence will affect the variance, the confidence interval is inflated through division of the variance (classical calculation for a proportion) by the square of the Youden index $(\mathrm{J}=\mathrm{Se}+\mathrm{Sp}-1) .22$ Additionally, the stochastic variability of the estimated Se and Sp (based on the size of the positive and negative population, respectively, used by Köhler et al. ${ }^{19}$ for test validation) is incorporated.

\section{Cluster test}

The spatial scan statistic ${ }^{23,24}$ was used to assess spatial clustering. A Bernoulli model was chosen, no overlapping was allowed, the scan window was defined as circular and, as recommended by Kulldorff et al.,25 the maximum window size was set to capture up to $50 \%$ of the events because clusters of excess risk that are larger than $50 \%$ of the events would be better viewed as cluster with lower risk outside the scanning window.

\section{Investigation into risk factors}

The association between age and herd size and sero-positivity for paratuberculosis was estimated jointly using logistic regression in order to account for potential mutual confounding. Animals were grouped in age classes 24-36 (202 heads of cattle), 37-48 (225), 49-60 (150), 61-72 (138) and $>72$ (181) months of age and herd size classes (defined by the number of animals eligible for testing $>24$ month) 1-100 (170 heads), 101-200 (219), 201-400 (256) and >400 (251) heads of cattle.

\section{Results}

Out of the 1001 samples, (instead of 1000 as specified above; a rounding effect in geographical stratification) desired for inclusion in the study and actually marked for paratuberculosis testing by the LIMS, only 896 samples (originating from herds with size ranging from 1 to 1482 eligible animals) were eventually examined. This was due to severe shortage in personnel at the LAV during the study but did not affect the desired precision of the estimate appreciably (see Sampling design section and confidence limits of the estimate below). Thirty-eight of the samples tested positive for antibodies against paratuberculosis (Cut-Off for test-positive sera: $\geq 70 \%$, according to the test manual and the test validation of Köhler $e t$ al. ${ }^{19}$ ). Thus, the apparent prevalence was $4.2 \%$ from which, taking into account the test accuracy, the true prevalence was estimated to $6.7 \%$ (95\% CI, 3.0-10.4\%). The geographical origin of the samples and their results are shown in Figure 1. To resolve individual samples from the same location, the sample origins are arbitrarily placed in the respective municipality. 
Sample size corresponds well with the underlying size of the target population and visual inspection does not provide evidence for clustering of positive results. The latter was confirmed by the spatial scan statistic which did not indicate any clusters. The calculation of stratum specific true prevalences for age classes (Figure 2) revealed a trend of increasing prevalences with age peaking at age class 5 (years) and then decreasing again. Prevalences also increased with herd size classes (Figure 3), slightly decreasing again for herd sizes $>400$ animals eligible for testing. Logistic regression indicated a significant effect on the true prevalence only for the age class of 5 years (Odds ratio: 5.2 (CI95 1.42, 19.02); $\mathrm{P}=0.013)$.

\section{Discussion}

Nielsen and Toft have critically reviewed the paratuberculosis studies in farmed animals in Europe from 34 publications between 1990 and April 2007.26 Among these were 30 studies aiming at the estimation of animal level prevalences in cattle. The authors gave critical comments to most of the latter studies, concluding that there is a continuing need for welldesigned studies of the prevalence of MAP infections. Major points of criticism were the fact that the sample tested frequently did not reflect the target population (study population/sampling frame not representative for target population, non-random sampling) and the lack of knowledge of the test accuracy of the diagnostic test used (i.e. no or unlikely true prevalences were calculated).

The study population of the presented study is probably well representing the target population because cattle registration with the national cattle database is obligatory, plausibility checked within the database and controlled by veterinary authorities. Simple random sampling using a formal randomization process was used to select samples from the sampling frame. In order to improve spatial representativeness the sample was geographically stratified. Thus, though only 896 of the 1001 samples intended for inclusion in the study were actually examined, spatial representativeness was very good (Figure 1). Representativeness of the sample with respect to age and herd size was assessed by class-wise comparison with the target population. It was considered as good (age, Figure 4) and very good (herd size, Figure 5), respectively. The apparent prevalence of $4.2 \%$ is very close to the apparent prevalence reported by Donat et al. for Saxony (4.4\%), a federal state adjacent to SaxonyAnhalt. 16 The true prevalence of $6.7 \%$ found in the presented study for the female cattle of Saxony-Anhalt indicates that the disease is present and may not be considered negligible.

The study was neither intended nor designed to investigate into risk factors and geographical distribution of paratuberculosis in female cattle of Saxony-Anhalt. But the

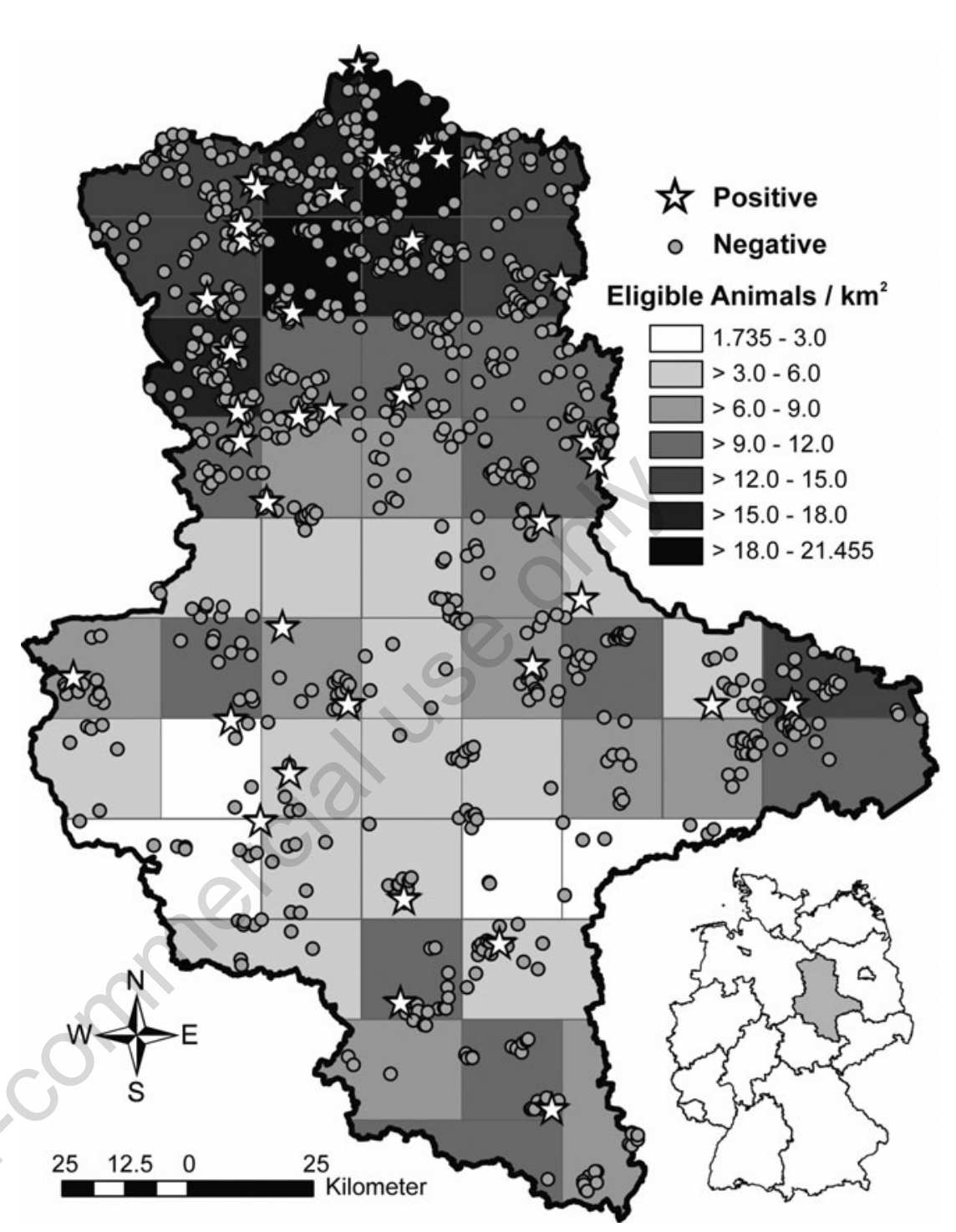

Figure 1. Geographical origin of test samples and results.

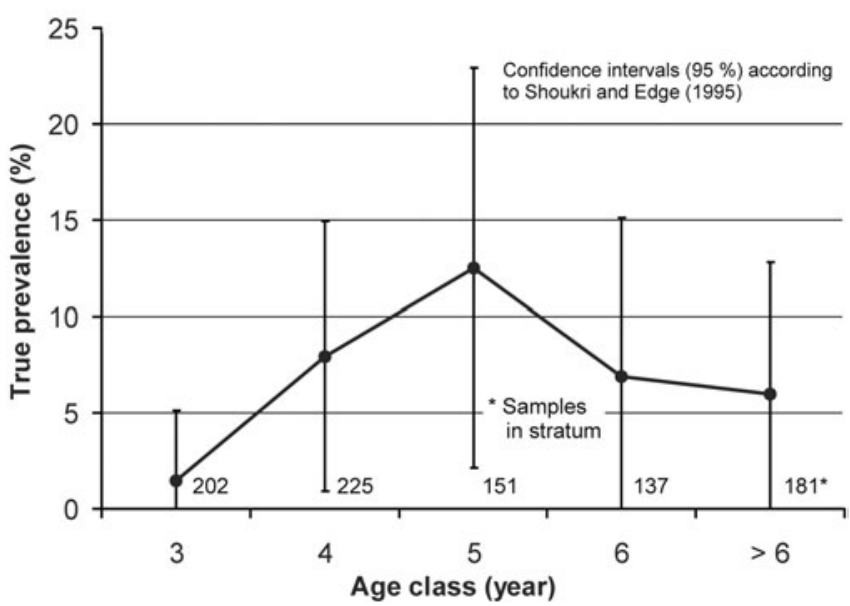

opportunity was taken to gain some insight test results was detected. But the power of the cluster test was low because of the small sample size and the imperfection of the test. As in into these aspects. No clustering of positive

Figure 2. Prevalence estimates for different age strata. 
the spatial scan statistic in logistic regression, test results were also assumed to be definite (perfect test assumption). Under this assumption a significant effect of age on the prevalence, which was adjusted for herd size, was indicated. But taking into account the imperfect nature of the test in confidence interval calculation reveals that intervals are overlapping and that no significance but only a trend can be demonstrated for the effect of age, as well as herd size. A similar effect of age was described by Nielsen and Ersbøll.20 According to them, prevalences in young animals are low because they still have to develop antibodies, most animals have a progression of the infection in the age interval 2.2-4.4 years of age and older animals are characterized by lower prevalences again because they show the socalled healthy worker survivor effect ${ }^{27}$ which means that healthy specimen accumulate in higher age classes because the diseased have already left the population. The positive effect
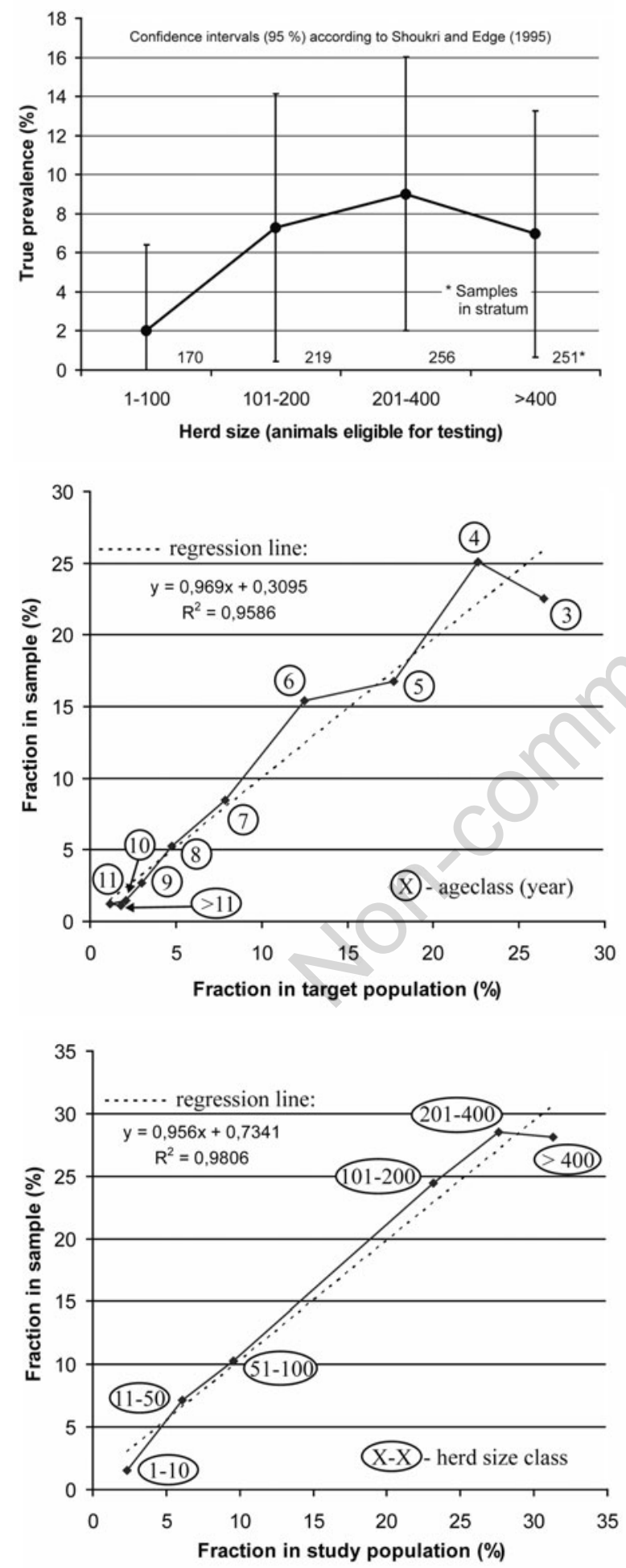

Figure 3. Prevalence estimates for different herd size strata.

of herd size on prevalence was also described by Woodbine et al. ${ }^{28}$ It might be explained by the growing number of trade contacts with herd size and therefore increasing risk of disease introduction.

Most particularly, because of the non-availability of reasonable but reliable mass testing strategies, to determine the paratuberculosis status of individual animals, no disease control program is presently planned in SaxonyAnhalt. But the awareness of dimension and distribution of disease occurrence is supposed to further improve the farmers' efforts on compliance with the Guidelines on Paratuberculosis published by the Federal Ministry of Food, Agriculture and Consumer Protection (BMELV) of Germany. The latter primarily refer to hygienic measures which reduce the risk of transmission of paratuberculosis within and between herds of cattle.

\section{References}

1. Stehman SM. Johne's disease (paratuberculosis). In: B. Smith (ed.) Large Animal Internal Medicine, pp 823-829. St. Louis, The C.V. Mosby Co., 1990.

2. Ott SL, Wells SJ, Wagner BA. Herd-level losses associated with Johne's disease on US dairy operations. Prev Vet Med 1999;40:179-92.

Figure 4. Representativeness with respect to age/class-wise comparison of fractions in target population and sample.

3. Dalziel TK. Chronical interstitial enteritis. Br Med J 1913;2:1068-70.

4. Hermon-Taylor J, Bull T. Crohn's disease caused by Mycobacterium avium subspecies paratuberculosis: a public health tragedy whose resolution is long overdue. J Med Microbiol 2002;51:3-6.

5. Greenstein RJ. Is Crohn's disease caused by a mycobacterium? Comparisons with leprosy, tuberculosis, and Johne's disease. Lancet Infect Dis 2003;3:507-14.

6. Selby WS. Mycobacterium avium subspecies paratuberculosis bacteraemia in patients with inflammatory bowel disease. Lancet. 2004;364:1013-14.

Figure 5. Representativeness with respect to herd size / class-wise comparison of fractions in target population and sample.

7. Shanahan F. Crohn's disease. Lancet 2002;359:62-9.

8. Feller M, Huwiler K, Stephan R, et al. Mycobacterium avium subspecies paratuberculosis and Crohn's disease: a systematic review and meta-analysis. Lancet Infect Dis 2007;7:607-13.

9. Abubakar I, Myhill D, Aliyu SH, Hunter PR. Detection of Mycobacterium avium subspecies paratuberculosis from patients with Crohn's disease using nucleic acidbased techniques: a systematic review and meta-analysis. Inflamm Bowel Dis 2008; 14:401-10.

10. Shanahan F, O'Mahony J. The mycobacteria story in Crohn's disease. Am J 
Gastoenterol 2005;100:1537-8.

11. Sartor RB. Does Mycobacterium avium subspecies paratuberculosis cause Crohn's disease? Gut 2005;54:896-8.

12. Behr MA, Kapur V.The evidence for Mycobacterium paratuberculosis in Crohn's disease. Curr Opin Gastroenterol 2008;24:17-21.

13. Donaghy JA, Linton M, Patterson MF, Rowe MT. Effect of high pressure and pasteurization on Mycobacterium avium ssp. paratuberculosis in milk. Lett Appl Microbiol 2007;45:154-9.

14. Grant IR, Ball HJ, Neill SD, Rowe MT. Inactivation of Mycobacterium paratuberculosis in cow's milk at pasteurisation temperatures. Appl Environ Microbiol. 1996;62:631-6.

15. Grant IR, Williams AG, Rowe MT, Muir DD. Efficacy of various pasteurisation timetemperature conditions in combination with homogenisation on inactivation of Mycobacterium avium subsp. paratuberculosis in milk. Appl Environ Microbiol 2005;71:2853-61.

16. Donat K, Eulenberger K, Kampfer P.
Blutserologische Untersuchungen zur Verbreitung von Mycobacterium avium spp. Paratuberculosis in sächsischen Rinderbeständen. Tieraerztl Umschau 2005;60:497-501.

17. Shoukri MM, Edge VL. Statistical methods for health sciences. Boca Raton, CRC Press, 1995.

18. Cochran WG. Sampling techniques. New York, Wiley, 1977.

19. Köhler H, Burkert B, Pavlik I, et al. Evaluation of five ELISA test kits for the measurement of antibodies against Mycobacterium avium subspecies paratuberculosis in bovine serum. Berl Munc Tierarztl Wochenschr 2008;121:203-10.

20. Nielsen SS, Ersbøll AK. Age at occurrence of Mycobacterium avium subsp. paratuberculosis in naturally infected dairy cows. $\mathrm{J}$ Dairy Sci 2006;89:4557-66.

21. Rogan WJ, Gladen B. Estimating prevalence from results of a screening test. Am J Epidemiol 1978;107:71-6.

22. Youden D. Index for rating diagnostic tests. Cancer 1950;3:32-5.

23. Kulldorff M, Nargawalla N. Spatial disease clusters: detection and inference. Stat Med 1995;14:799-810.

24. Satscan.org [Internet]. Boston, Kulldorff M, Information Management Services Inc., c2005 [updated 2011 March 9; cited 2011 Sept 12] Available from: http://www. satscan.org/

25. Kulldorff M, Athas WF, Feurer EJ, et al. Evaluating cluster alarms: a space-time scan statistic and brain cancer in Los Alamos, New Mexico. Am J Public Health 1998;88:1377-80.

26. Nielsen SS, Toft N. A review of prevalences of paratuberculosis in farmed animals in Europe. Prev Vet Med 2009;88:1-14.

27. Arrighi HM, Hertz-Picciotto I. The evolving concept of the healthy worker survivor effect. Epidemiology 1994;5:189-96.

28. Woodbine KA, Schukken YH, Green LE, et al. Seroprevalence and epidemiological characteristics of Mycobacterium avium subsp. paratuberculosis on 114 cattle farms in south west England. Prev Vet Med 2009;89:102-9. 